\title{
NUCLEATION AND SPINODAL DECOMPOSITION IN TERNARY-COMPONENT ALLOYS
}

WILL HARDESTY AND COLLEEN ACKERMANN

Date: July 28, 2009

Dr. Thomas Wanner and Dr. Evelyn Sander. 
Abstract. put abstract here

\title{
NUCLEATION AND SPINODAL DECOMPOSITION IN TERNARY-COMPONENT ALLOYS
}

\author{
WILL HARDESTY AND COLLEEN ACKERMANN
}

1. INTRODUCTION

Alloys are composite materials which are formed by mixing a number of pure metals together at a high temperature and then rapidly quenching or cooling the mixture to form a solid. During the process of quenching, the components undergo a phase seperation in which they begin to form patterns. These pattern formations can be divided into two classes: Nucleation and Spinodal Decomposition. Qualitatively nucleation occurs when the individual components begin to materialize from the homogenous mixture as isolated droplets or bubbles. Spinodal decomposition occurs when the components form connected snakelike patterns. This behavior is modelled mathematically by the Cahn-Moral equation.

\section{Background and Research Methods}

\subsection{Cahn-Moral system.}

2.1.1. The equations. The Cahn-Morral equation is given by,

$$
\begin{array}{rcc}
u_{t}=-\Delta\left(\varepsilon^{2} \Delta u+f(u)\right) & \text { on } & \Omega \\
\frac{\partial u}{\partial \nu}=\frac{\partial \Delta u}{\partial \nu}=0 & \text { on } & \partial \Omega .
\end{array}
$$

Where the energy of the system is modelled by the Van der Waals free energy functional,

$$
E_{\varepsilon}[u]=\int_{\Omega}\left(\frac{\varepsilon^{2}}{2} \cdot|\nabla u|^{2}+F(u)\right) d x
$$

Date: July 28, 2009.

Dr. Thomas Wanner and Dr. Evelyn Sander. 
The term $-f$ is defined as the derivative of the double-well potential, $F$ from the free energy functional. The domain $\Omega \in \mathbb{R}=[0,1]$, with imposed Neumann boundary conditions.

\subsubsection{The double well potential.}

2.2. Gibbs Simplex. The gibbs simplex is defined as:

$$
\mathcal{G}=\left\{(u, v, w) \in \mathbb{R}^{3}: u+v+w=1, u \geq 0, v \geq 0, w \geq 0\right\}
$$

$\mathcal{G}$ represents the set of all possible states or average mass concentrations of the ternary alloy, this follows from the conservation of mass. The Gibbs simplex can be divided into regions corresponding to nucleation and spinodal decomposition, which are determined through linearisation analysis.

Given a state $(\bar{u}, \bar{v}, \bar{w})$, the stability of that particular state is given by computing the eigenvalues of $J_{f}(\bar{u}, \bar{v}, \bar{w})$, where $J_{f}$ is the Jacobian of $f(u)$ [4]. The state $(\bar{u}, \bar{v}, \bar{w})$ is in the nucleation region if $J_{f}$ has no positive eigenvalues otherwise it lies in the spinodal region [2]. These regions can be depicted graphically with the Gibbs Triangle, where each color represents a different region.

2.3. The nonlinearities. In our research we used two nonlinearities. A quadratic nonlinearity:

$$
F(u, v, w)=\frac{u^{2} v^{2}+\left(u^{2}+v^{2}\right)\left(w^{2}\right)}{4}
$$

And a logarithmic nonlinearity:

$$
F(u, v, w)=3.5(u v+u w+v w)+u \ln u+v \ln v+w \ln w
$$

The nonlinearities were chosen so that they were double well potentials and symmetric. Symmetric means that each componenet contributes to the nonlinearity in the same way making invesigations simpler.

Both of the nonlinearities have been investigated previously in both the one and two dimensional cases. However, most of the previous research was done with time 
variation. Our research differs in that we are looking at solutions at one moment in time and comparing results from different nonlinearities.

\section{Results And Discussion}

3.1. Gibbs Triangle. For each of our nonlinearities we created Matlab code which shows which regions on the Gibbs simplex have no, one or two positive eigenvalues. In the pictures below the Gibbs simplex is projected onto the plane.

The red area represents the nucleation region where there are no positive eigenvalues. The light blue and dark blue represent the regions where there are one and two positive eigenvalues respectively. Both areas are considered part of the spinodal region.

3.2. Path following. Our ultimate goal in path following was to trace paths in the nucleation region. However, the secondary branches in the nucleation region are not connected to the trivial branch. To reach the non-trivial nucleation branches we had to begin in the spinodal region. First we varied $\lambda=\frac{1}{\varepsilon^{2}}$. to get onto the second secondary branch in the spinodal region. After following the second branch and it's bifurcations we attempted to follow in $\alpha=\frac{\bar{u}+\bar{v}}{2}$ back into the nucleation region. Finally we varied $\beta=\frac{\bar{u}-\bar{v}}{2}$ in the nucleation region.

3.2.1. Tracing the quadratic nonlinearity.

\subsubsection{Tracing the logarithmic nonlinearity.}

\subsection{Troubleshooting.}

\section{Conclusions And Future Work}

We would like to successfully trace alpha back into the nucleation region from several branches at the same $\lambda$ value and compare bubble formations. We hope to eventually find which bubble formations are possible at each value of $\lambda$ for each nonlinearity. Our third partner James O'Beirne will be investigating a sixth degree polynomial nonlinearity and comparing the results with our investigations. Eventually the research should be expanded to two and three dimensions to see if 
results vary significantly. Also more than three components will components will be used. Both of these extensions though requiring more computing power, will make the model considerably more reliable.

- higher dimensional domains

- more than 3 components

- different nonlinearities

\section{AcKnowledgements}

Thanks to Dr. Thomas Wanner, Dr. Evelyn Sander, James O'beirne the Department of Defense, the National Science Foundation, and the Deparment of Mathematical Sciences at George Mason University.

\section{REFERENCES}

[1] Kathleen T. Alligood, Tim D. Sauer, and James A. Yorke, Chaos, An Introduction to Dynamical Systems, Springer- Verlag New York, Inc., New York, New York, 1997.

[2] Jonathan P. Desi, Hanein H. Edrees, Joseph J. Price, Evelyn Sander, and Thomas Wanner, The Dynamics of Nucleation in Stochastic Cahn-Morral Systems (May 29, 2009).

[3] Junseok Kim and Kyungkeun Kang, A numerical method for the ternary Cahn-Hilliard system with a degenerate mobility, Applied Numerical Mathematics (May 9, 2009).

[4] Stanislaus Maier-Paape, Barbara Stoth, and Thomas Wanner, Spinodal Decomposition for Multicomponent Cahn-Hilliard Systems, Journal of Statistical Physics (September 21, 1999).

Department of Mathematical Sciences, George Mason

E-mail address: hardes1@umbc.edu

E-mail address: cackerm@vt.edu 